\title{
Experimental study on the protective effects of edaravone against ischemic spinal cord injury
}

\author{
Kazuchika Suzuki, MD, ${ }^{a}$ Teruhisa Kazui, MD, PhD, ${ }^{a}$ Hitoshi Terada, MD, PhD, ${ }^{a}$ Kazuo Umemura, MD, PhD, \\ Yasuhiko Ikeda, MD, PhD, ${ }^{\mathrm{b}}$ Abul Hasan Muhammad Bashar, MBBS, PhD, ${ }^{\text {a }}$ Katsushi Yamashita, MD, PhD, ${ }^{a}$ \\ Naoki Washiyama, MD, PhD, ${ }^{a}$ Takayasu Suzuki, MD, ${ }^{a}$ Kazuhiro Ohkura, MD, PhD, and Junji Yasuike, MDa
}

Objective: Reactive free radical species are thought to be involved in ischemic spinal cord injury. We investigated the effects of edaravone (Mitsubishi Pharma Co, Tokyo, Japan), a free radical scavenger, on spinal ischemia-reperfusion injury in a rabbit model. We also sought to estimate free radicals in the spinal cord using the microdialysis method.

Methods: Spinal cord ischemia was induced in New Zealand White rabbits. The animals were then divided into 4 groups. In the first experiment, which was carried out in group A (non-edaravone treated) and group B (edaravone treated), we assessed neurologic function and evaluated spinal cord histopathology. In the second experiment, which was performed in group C (non-edaravone treated) and group D (edaravone treated), we sequentially estimated the level of free radical species in the spinal cord with the microdialysis method.

Results: In the first experiment group B showed better neurologic function than group $\mathrm{A}$. The number of viable neurons in the spinal cord gray matter was also higher in group B than in group A. The second experiment revealed that the level of free radical species was lower in group D at 75, 90, and 150 minutes after the beginning of reperfusion compared with levels seen in group $\mathrm{C}$. The appearance of free radical species in the latter group was found to have a biphasic pattern, with peaks at 75 and 150 minutes after the beginning of reperfusion.

Conclusion: Edaravone exerted a significant protective effect on the spinal cord against ischemia-reperfusion injury by suppressing the level of free radical species, which was demonstrated with the microdialysis method.

The First Department of Surgery and The Department of Pharmacology, ${ }^{\mathrm{b}}$ Hamamatsu University School of Medicine, Hamamatsu, Japan.

Received for publication July 1, 2005; revisions received Aug 19, 2005; accepted for publication Aug 26, 2005.

Address for reprints: Kazuchika Suzuki, MD, The First Department of Surgery, Hamamatsu University School of Medicine, 1-20-1 Handayama, Hamamatsu, 4313192, Japan (E-mail: kazuchi@fj9.so-net. ne.jp).

J Thorac Cardiovasc Surg 2005;130:1586-92 $0022-5223 / \$ 30.00$

Copyright (C) 2005 by The American Association for Thoracic Surgery

doi:10.1016/j.jtcvs.2005.08.049
$\mathrm{P}$ araplegia or paraparesis resulting from spinal cord ischemia during descending or thoracoabdominal aortic aneurysm surgery is a dreaded result. Various methods have been used for the prevention of ischemic spinal cord injury, such as systemic or topical cooling, distal aortic perfusion, intercostal artery reimplantation, cerebrospinal fluid drainage, and pharmacologic agents. ${ }^{1-3}$ Intraoperative monitoring of spinal cord function, such as motor evoked potential and sensory evoked potential, has also been in clinical use for many years. ${ }^{4,5}$ Although neurologic deficits have significantly decreased in recent times because of the use of these adjuncts, the incidence of paraplegia is still high at $6.6 \%$ to $8.3 \%$ in the patients with extent II thoracoabdominal aortic aneurysms. ${ }^{1-3,6}$ With a view to further improving spinal cord protection, a number of pharmacologic agents with varying mechanisms of action have been brought under experimental evaluation and clinical trial. Earlier, we reported our experimental study on spinal cord protection with $N$-methyl-D-aspartate receptor antagonist (dextrorphan) and $\mathrm{Na}-\mathrm{Ca}^{2+}$ channel blocker (NS-7) ${ }^{7,8}$ Unfortunately, these chemicals are not in clinical use because of their unacceptable 


\section{Abbreviations and Acronyms \\ $\mathrm{CNS}=$ central nervous system \\ HPF $=2$-[6-(4'-hydroxy)phenoxy-3H-xanthen-3-on-9-yl $]$ benzoic acid}

side-effect profile. With an aim to find a clinically applicable pharmacologic agent, we directed our attention toward the free radical hypothesis of central nervous system (CNS) ischemic injury and a way to prevent it. ${ }^{9}$ In this connection we considered the potential of edaravone (Mitsubishi Pharma Co, Tokyo, Japan), a commercially available free radical scavenger, the cerebral protective effects of which have been demonstrated both experimentally and clinically. ${ }^{10,11} \mathrm{We}$ thought that the spinal cord, a part of the CNS, could also be protected from ischemic injury by edaravone. In the present study we assessed motor function status, as well as spinal cord histopathology, to evaluate the effect of prophylactically administered edaravone on the rabbit spinal cord after ischemiareperfusion injury. Additionally, we sequentially estimated the level of free radicals within the spinal cord by using the microdialysis method to document the effect of edaravone.

\section{Materials and Methods}

\section{Animal Care and Surgical Procedure}

We used male New Zealand white rabbits weighing 2.5 to $3.5 \mathrm{~kg}$. All animals received humane care in compliance with the "Guide for the care and use of laboratory animals" prepared by the Institute of Laboratory Animal Resources, National Research Council, and published by the National Academy Press, revised 1996, and the "Guidelines for animal experimentation" of Hamamatsu University School of Medicine.

\section{Creating a Spinal Cord Ischemia Model}

The animals were anesthetized with intravenous sodium pentobarbital $(25 \mathrm{mg} / \mathrm{kg})$. A continuous intravenous infusion line was secured at the marginal ear vein with a 24-gauge catheter. An arterial line was secured in the left common carotid artery with a 24-gauge catheter to monitor the proximal arterial pressure. Rectal temperature was continuously monitored with a flexible probe. An electric blanket (Sharp Co, Osaka, Japan) was used for retaining warmth, so that any decrease in temperature would not exceed $1^{\circ} \mathrm{C}$. A $4 \mathrm{~F}$ balloon-tipped catheter (Goodtec Inc, Huntington Beach, Calif) was inserted through an arteriotomy in the left femoral artery into the abdominal aorta for a distance of about $15 \mathrm{~cm} .{ }^{12}$ Preliminary investigations, including laparotomy, confirmed that the balloon came to lie 0.5 to $1.2 \mathrm{~cm}$ distal to the left renal artery. After intravenous administration of heparin $(100 \mathrm{U} / \mathrm{kg})$, the balloon was inflated to obstruct the aortic blood flow. Complete cessation of blood flow was confirmed by a pressure decrease in the abdominal aorta measured with the help of a pressure sensor attached at the distal end of the balloon. In this study we applied a 30-minute obstruction of the abdominal aorta. A total of 30 animals underwent spinal cord ischemia-reperfusion injury. The animals were then randomly divided into 4 groups. The first experiment was carried out in groups $A(n=10)$ and $B(n=10)$, with only the latter receiving intravenous edaravone. The rabbits of both groups were assessed for hind-limb motor function 24 and 48 hours after the beginning of reperfusion. They were then killed, and their lumbar spinal cords were removed for histopathologic examination. In the second experiment (groups C and D) we applied the microdialysis method to estimate the level of free radical species within the spinal cord. Animals in group D $(\mathrm{n}=$ $5)$ received edaravone, whereas those in group $C(n=5)$ did not.

\section{Drug Delivery}

In groups B and D intravenous edaravone was continuously delivered at a rate of $2.5 \mathrm{mg} / \mathrm{kg} / \mathrm{h}$ with an infusion pump (Terumo, Tokyo, Japan). The dosage was decided on the basis of previous studies that report the effective dose of edaravone to be 1.5 to $3.0 \mathrm{mg} / \mathrm{kg}$ in cerebral ischemic rat models. ${ }^{13,14} \mathrm{We}$ started administration of the drug 30 minutes before the obstruction of blood flow because according to a previous report, a steady plasma level of edaravone was obtained 30 minutes after the beginning of intravenous injection. ${ }^{15}$ Because it is not clear when the influence of free radicals on spinal tissue disappears, we performed continuous administration of edaravone until 180 minutes after the beginning of reperfusion. In the non-edaravone-treated groups (groups A and C) normal saline solution was delivered intravenously under similar conditions as in the edaravone-treated groups.

\section{Microdialysis Method}

In the second experiment animals underwent tracheostomy in the supine position and were then turned to the prone position. Vecuronium bromide $(0.1 \mathrm{mg} / \mathrm{kg})$ was administered as a muscle relaxant to avoid movement while doing laminectomy, and mechanical ventilation was started. The vertebral canal was opened at the level of L3. The dura mater was removed, and the spinal cord was exposed. A microdialysis probe with a membrane length of $2 \mathrm{~mm}$ (CMA Microdialysis Inc, North Chelmsford, Mass) was inserted into the spinal cord and stabilized. The probe was connected to a microinjection pump with fine microdialysis catheters (CMA Microdialysis Inc). In this study 2-[6-(4'-hydroxy)phenoxy-3H-xanthen-3-on-9-yl]benzoic acid (HPF; Daiichi Pure Chemicals Co, Tokyo, Japan) solution was used as perfusate. HPF becomes strongly fluorescent when it reacts with hydroxyl radical or peroxynitrite (Figure 1). ${ }^{16}$ Free radicals produced in the spinal tissue react with the HPF through the microdialysis membrane. HPF solution, which was diluted to a concentration of $50 \mu \mathrm{mol} / \mathrm{L}$, was perfused at a rate of $2 \mu \mathrm{L} / \mathrm{min}$ until 180 minutes after the beginning of reperfusion. HPF was collected every 15 minutes after it had passed through the microdialysis circuit. It was then measured for its fluorescence intensity with a spectrofluorometer (Japan Spectroscopic Co, Tokyo, Japan) at $515 \mathrm{~nm}$, with excitation at 490 $\mathrm{nm}$. Higher intensity of fluorescence meant a higher level of free radicals. 
<smiles>O=C([O-])c1ccccc1-c1c2ccc(=O)cc-2oc2cc(Oc3ccc(O)cc3)ccc12</smiles>

almost non-fluorescent

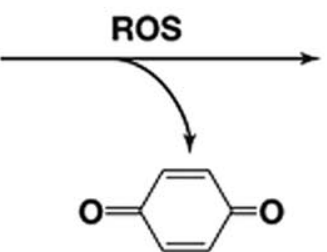

strongly fluorescent

Figure 1. The putative reaction scheme of 2-[6-(4'-hydroxy)phenoxy-3H-xanthen-3-on-9-yl]benzoic acid (HPF). HPF, which is almost completely nonfluorescent, is o-dearylated on reaction with reactive oxygen species (ROS) to yield strongly fluorescent fluorescein.

\section{Neurologic Evaluation}

Neurologic status was scored on the basis of assessment of hindlimb motor function 24 and 48 hours after reperfusion in the first experiment according to the Johnson neurologic recovery scale ${ }^{17}$ : 0 , hind-limb paralysis; 1 , severe paraparesis; 2 , functional movement but no hopping; 3, ataxic hopping; 4, minimal ataxia; 5, normal.

\section{Histopathologic Examination}

After neurologic evaluation, the animals in the first experiment were killed with an overdose of intravenous sodium pentobarbital. The lumbar spinal cord was quickly removed and fixed overnight in $10 \%$ buffered formalin. Paraffin-embedded sections were processed for hematoxylin and eosin staining, and histopathologic evaluation of spinal cord tissue from the L3 segment was performed under a light microscope. The examination included a count of the viable neurons per section in the gray matter.

\section{Statistical Analysis}

Statistical analysis of the neurologic recovery score was performed with the nonparametric Mann-Whitney $U$ test. Statistical analyses of the mean proximal arterial pressure, rectal temperature data, and numbers of the remaining neurons were done with the unpaired $t$ test. In the analyses of fluorescence intensity, the unpaired $t$ test was used for comparison between groups C and D, whereas the paired $t$ test was used for comparison within group C.

\section{Results}

\section{Blood Pressure and Rectal Temperature}

Mean proximal arterial pressures and the lowest rectal temperatures are shown in Table 1. In both experiments there were no differences in mean proximal aortic pressures and rectal temperatures between the edaravone-treated and nonedaravone-treated groups.

\section{Neurologic Evaluation}

Neurologic status is shown in Figure 2. In group $A$ the Johnson neurologic recovery score was 0 (hind-limb paralysis) in all animals at 24 and 48 hours after the beginning of reperfusion. In group B the scores at 24 hours after the beginning of reperfusion were as follows: 5 in 3 animals, 4 in 1 animal, 3 in 1 animal, and 0 in 2 animals, with an average score of $3.4 \pm 1.8$. At 48 hours after the beginning of reperfusion, the scores were as follows: 5 in 5 animals, 4 in 1 animal, 3 in 1 animal, and 0 in 2 animals, with an average of $3.3 \pm 2.2$. In comparison with group $\mathrm{A}$, animals

TABLE 1. Mean proximal arterial pressure and lowest rectal temperature during obstruction of the abdominal aorta

\begin{tabular}{lcccccc}
\hline & $\begin{array}{c}\text { Before ischemia } \\
(\mathbf{m m ~ H g})\end{array}$ & $\begin{array}{c}\text { During ischemia } \\
(\mathbf{m m ~ H g})\end{array}$ & \multicolumn{3}{c}{ After beginning of reperfusion: } & \multicolumn{2}{c}{$\begin{array}{c}\text { Lowest rectal } \\
\text { temperature }\left({ }^{\circ} \mathbf{C}\right)\end{array}$} \\
\hline Group A & $107 \pm 10$ & $118 \pm 14$ & $103 \pm 15$ & $110 \pm 12$ & $112 \pm 10$ & $37.9 \pm 0.5$ \\
Group B & $116 \pm 6$ & $121 \pm 6$ & $110 \pm 10$ & $122 \pm 11$ & $116 \pm 7$ & $38.1 \pm 0.9$ \\
Group C & $118 \pm 2$ & $120 \pm 7$ & $110 \pm 13$ & $115 \pm 11$ & $111 \pm 10$ & $38.1 \pm 0.2$ \\
Group D & $118 \pm 9$ & $121 \pm 5$ & $107 \pm 11$ & $117 \pm 9$ & $118 \pm 6$ & $38.3 \pm 0.4$ \\
\hline
\end{tabular}

Values represent means \pm standard deviation. Groups $A(n=10)$ and $C(n=5)$ underwent spinal ischemia alone. Groups $B(n=10)$ and $D(n=5)$ received continuous intravenous infusion of edaravone at the rate of $2.5 \mathrm{mg} / \mathrm{kg} / \mathrm{h}$. 


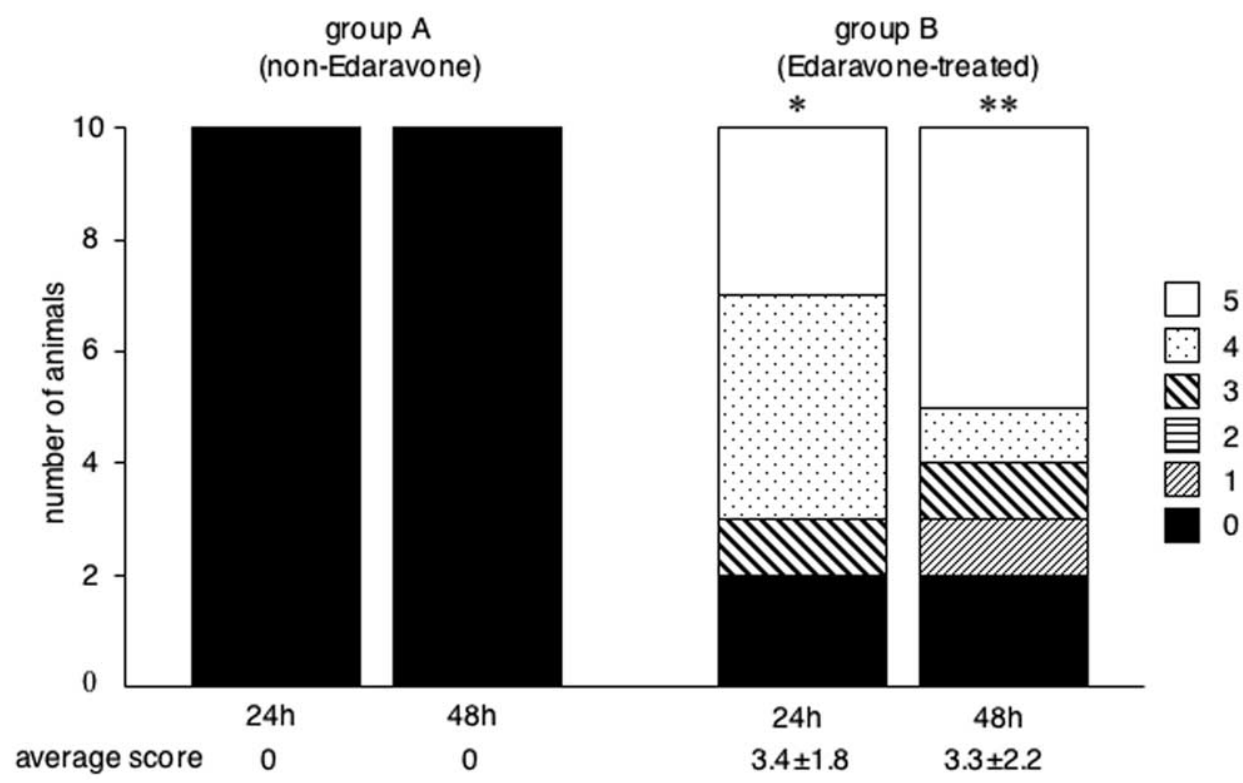

Figure 2. Neurologic status 24 and 48 hours after the beginning of reperfusion, as evaluated by using the Johnson recovery scale. Average scores represent means \pm standard deviation. Group B had significantly higher scores in comparison with group $A$ at 24 and 48 hours after the beginning of reperfusion (at 24 hours: ${ }^{*} P=.0025$ compared with group $A$; at 48 hours: ${ }^{* *} P=.0025$ compared with group $\left.A\right)$.

in group B had significantly higher scores $(P=.0025$ at 24 hours and $P=.0025$ at 48 hours). The neurologic scores in group B at 24 and 48 hours after the beginning of reperfusion were similar.

\section{Histopathologic Evaluation}

Histopathologic findings are shown in Figure 3. The lumbar spinal cords of rabbits with fully preserved neurologic function (score 5) after edaravone administration were histologically well preserved. The cross-sections of the spinal cord showed normal-looking motor neurons having clear nucleoli and Nissl substance (Figure 3, group B). On the other hand, spinal cord sections of rabbits with paralysis (score 0 ) demonstrated necrotic changes in the form of destruction and vacuolization of the gray matter, pyknosis of neurons, and eosinophilic changes of the cytoplasm (Figure 3, group A). The numbers of viable neurons in the gray matter were $20.0 \pm$ 10.8 in group $\mathrm{A}$ and $81.5 \pm 27.2$ in group $\mathrm{B}(P=.008$, Figure 4). Histopathologic findings correlated well with neurologic functions in both the edaravone-treated and nonedaravone-treated groups.

\section{Microdialysis Method}

The fluorescence intensities of HPF are shown in Figure 5. The intensity in groups $\mathrm{C}$ and $\mathrm{D}$ at 75 minutes, 90 minutes, and 150 minutes after the beginning of reperfusion were $1341 \pm 69$ and $1225 \pm 27,1294 \pm 51$ and $1187 \pm 60$, and $1245 \pm 19$ and $1168 \pm 25$, respectively. The fluorescence intensities of HPF were significantly lower in group D at 75, 90 , and 150 minutes after the beginning of reperfusion compared with those in group C $(P=.0162, P=.00158$, and $P=.0012$, respectively). This means that the level of free radicals was lower in the edaravone-treated animals. Comparing the fluorescence intensity at 75 minutes after the beginning of reperfusion with those at 15, 30, and 45 minutes after the beginning of reperfusion in group $\mathrm{C}$, the intensities at 75 minutes were significantly higher $(P=$ $.0274, P=.0044$, and $P=.0019$, respectively). This means that the production of free radicals in the ischemic spinal cord began to increase between 60 and 75 minutes after the beginning of reperfusion. Although not statistically significant, the fluorescence intensity of HPF tended to show another peak between 150 and 165 minutes after the beginning of reperfusion in group $\mathrm{C}$.

\section{Discussion}

In this study we demonstrated that edaravone was effective in preventing motor function and histopathologic complications of spinal cord ischemia-reperfusion injury in rabbits. Earlier, Takahashi and colleagues ${ }^{18}$ applied edaravone in rabbit spinal ischemia models and documented its effectiveness by immunohistochemically assessing the levels of superoxide dismutase and nitric oxide synthatase, but the study did not demonstrate a direct relationship between the administration of edaravone and the decrease in the level of 

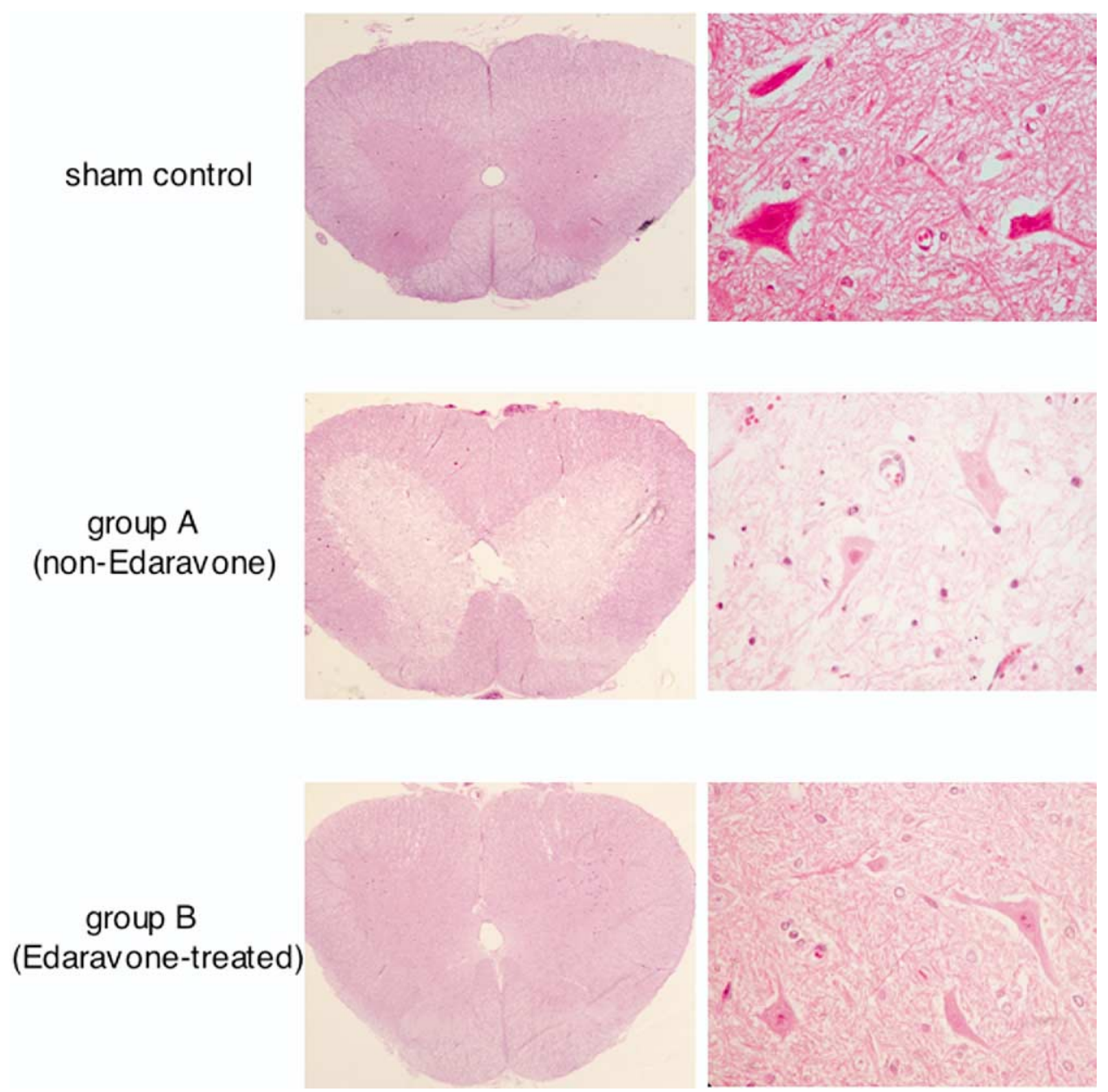

Figure 3. Histopathologic findings of the spinal cord sections stained with hematoxylin and eosin 48 hours after the beginning of reperfusion. Sections from each group were examined with 2 different magnifications. (Original magnification: left panel, $40 \times$; right panel, $400 \times$.) The spinal cord of sham control animals was intact. In group A spinal cords showed necrotic changes, including destruction and vacuolization of the gray matter. In group $B$ the gray matter was largely preserved, with normal-looking motor neurons.

free radicals. It is for the first time that we used the microdialysis method to document the production of free radicals within the spinal cord as a result of ischemia-reperfusion injury, which were scavenged by edaravone. It is interesting that the inhibition of free radical production by edaravone is directly demonstrable. Free radical species lead to oxidation of lipids, proteins, and nucleic acids, which might alter cellular function in a critical way, resulting in CNS injury and subsequent neurologic dysfunction. ${ }^{19,20}$ Normally, toxic free radical species are converted to harmless metabolites by superoxide dismutase or catalase. ${ }^{9,20}$ The oxidative damage potential and the antioxidant defense capacity of the body are in a dynamic equilibrium at all times. ${ }^{21}$ Once this equilibrium is disturbed, as in ischemic injury, there is excessive production of free radical species, which ultimately leads to an increase in the level of hydroxyl radical. ${ }^{21,22}$ Hydroxyl radical is one of the most reactive free radical species and is known to hydroxylate almost all components of the living cell, resulting in injury and cell death. ${ }^{23}$ In previous reports excessive levels of hydroxyl radical were documented in the damaged brain. ${ }^{24,25}$ As with ischemic brain injury, hydroxyl radical was also shown to increase in the regions of ischemia-reperfusion injury of the spinal cord. ${ }^{9,26}$ Because free radical species have a very short half-life, it is necessary to apply trapping agents, such as salicylic acid or phenylalanine, to detect them. However, the chemical compounds resulting from trapping of free radical species are very unstable substances. Moreover, it is difficult to accurately measure a very small quantity of these compounds. ${ }^{27} \mathrm{We}$ thought that it was possible to estimate even a small quantity of free radical species using the 


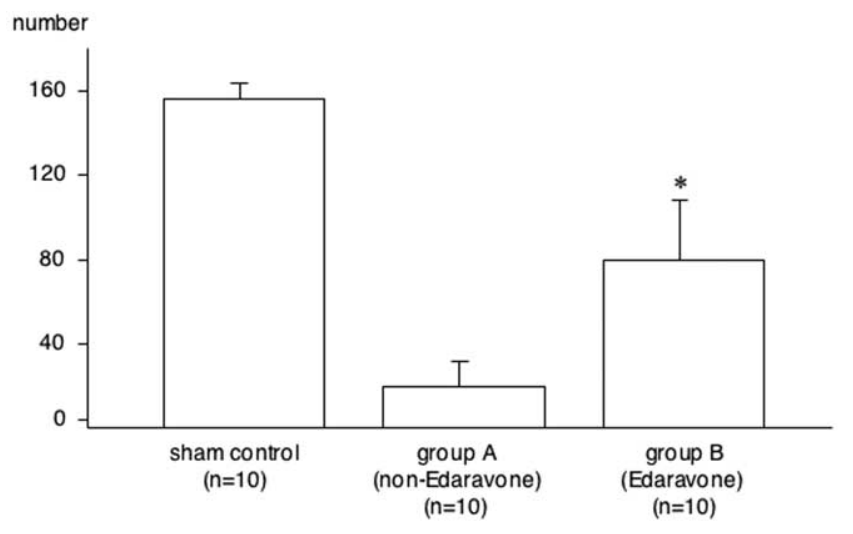

Figure 4. The number of viable neurons in the gray matter of lumbar spinal cord sections stained with hematoxylin and eosin. Group B had significantly more viable neurons than group $A\left({ }^{*} P=.008\right.$ compared with group A).

microdialysis method. The advantage of this method is that it allows for sequential evaluation of any fluctuation in the level of free radical species throughout the duration of ischemia and reperfusion of the spinal cord in a dynamic manner. In the second experiment we found that the fluorescence intensity peaked at 75 minutes after the beginning of reperfusion in the non-edaravone-treated group. This suggests that the production of free radical species was the highest between 60 and 75 minutes after the beginning of reperfusion. It also indicates that tissue injury in the spinal cord might be more severe a certain time after the commencement of reperfusion rather than immediately after it.
It is thought that the production of free radicals gradually increases as blood flow to the ischemic regions recovers and reaches a delayed peak. ${ }^{28}$ According to the findings of brain ischemia-reperfusion injury, the peak of free radical appearance varies between 15 and 40 minutes after reperfusion. $^{25,28}$ In our experiment the peak appeared to be more delayed. We think that the following factors might have caused this delay. The extent and severity of tissue injury experienced because of ischemia vary with experiments because of difference in ischemic time. Moreover, time required for the recovery of blood flow to ischemic regions differs between the brain and spinal cord because of the difference in the pattern of the collateral blood flow in the 2 regions. Although the production of free radicals was biphasic in our study, it is possible that the latter peak was caused by inflammatory cells, particularly neutrophils, which are a well-known source of several kinds of free radicals. $^{29}$ The level of free radicals in the edaravonetreated group was particularly lower in comparison with the non-edaravone-treated group at 75, 90, and 150 minutes after the beginning of reperfusion. It suggests that the appearance of free radical species was suppressed by administering edaravone. We think that the free radicals that were produced as a result of ischemia-reperfusion injury were scavenged by edaravone.

However, the findings of this experimental study should be interpreted bearing in mind its limitations. The spinal cord circulation in rabbits might not be quite the same as it is in human subjects. Drug toxicity of edaravone is another concern that is likely to limit its clinical application. Acute renal failure and increased liver transaminases have been

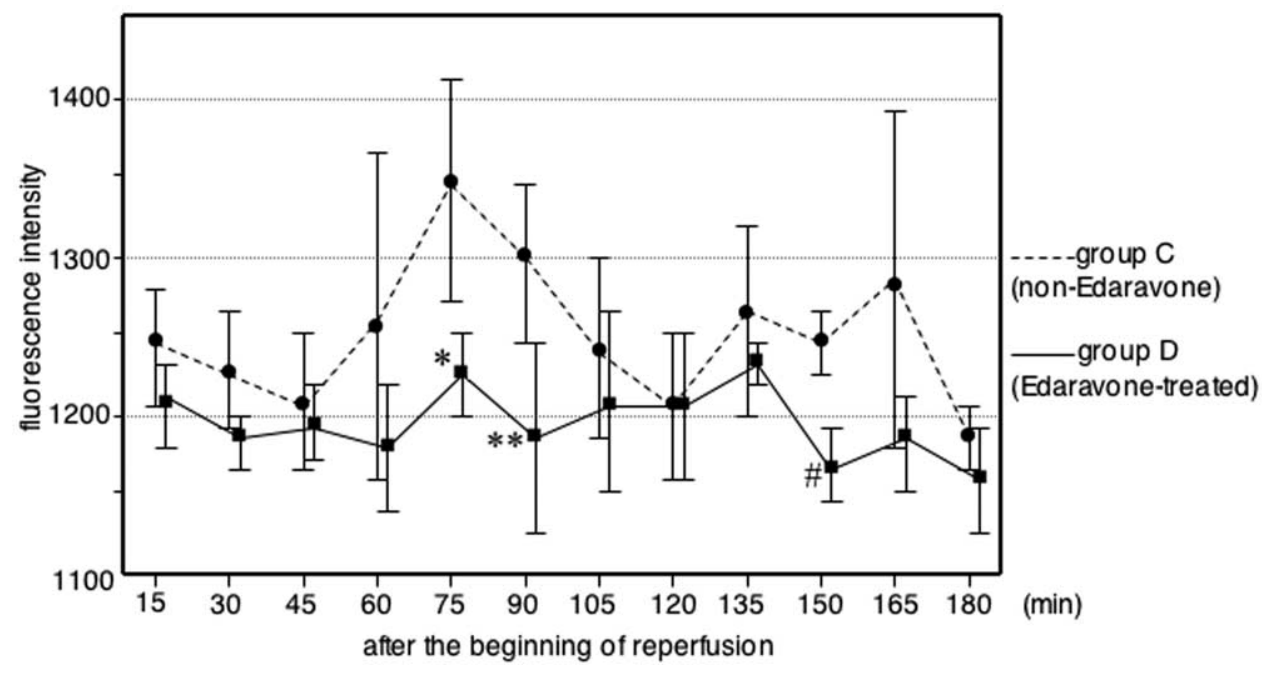

Figure 5. The fluorescence intensities of 2-[6-(4'-hydroxy)phenoxy-3H-xanthen-3-on-9-yl]benzoic acid at every 15 minutes from 15 to 180 minutes after the beginning of reperfusion. Intensities were significantly lower in group $D$ at 75,90 , and 150 minutes after the beginning of reperfusion than in group $C$ (at 75 minutes: ${ }^{*} P=.0162$; at 90 minutes: ${ }^{* *} P=.00158$; at 150 minutes: $\left.\# P=.0012\right)$. 
reported as possible complications in the clinical setting. ${ }^{30}$ Moreover, it should be emphasized that edaravone does not have any effect on the tissue injury experienced at the onset of ischemia. It only acts on the subsequent free radical injury caused at the time of reperfusion. Therefore complete neurologic recovery after spinal cord ischemiareperfusion injury should not be expected with the use of edaravone.

In conclusion, prophylactic administration of edaravone, a free radical scavenger, in a rabbit spinal ischemia model resulted in partial prevention of neurologic functional impairment, with an increase in the number of viable motor neurons in the gray matter. The microdialysis method with HPF was useful in evaluating the level of free radical species. With this method, it was possible to demonstrate that free radicals produced within the spinal cord as a result of ischemia-reperfusion injury were scavenged by edaravone. Prophylactic administration of edaravone might be a useful adjunct for spinal cord protection during descending or thoracoabdominal aortic operations.

\section{References}

1. Safi HJ, Miller CC III, Huynh TTT, Estrera AL, Porat EE, Winnerkvist AN, et al. Distal aortic perfusion and cerebrospinal fluid drainage for thoracoabdominal and descending thoracic aortic repair. Ann Surg. 2003;238:372-81.

2. Kouchoukos NT, Daily BB, Rokkas CK. Hypothermic bypass and circulatory arrest for operations on the descending thoracic and thoracoabdominal aorta. Ann Thorac Surg. 1995;60:67-77.

3. Acher CW, Wynn MM, Hoch JR, Popic P, Archibald J, Turnipseed WD. Combined use of cerebral spinal fluid drainage and naloxone reduces the risk of paraplegia in thoracoabdominal aneurysm repair. J Vasc Surg. 1994;19:236-46.

4. Galla JD, Ergin MA, Sadeghi AM, Lansman SL, Danton J, Griepp RB. A new technique using somatosensory evoked potential guidance during descending and thoracoabdominal aortic repairs. J Card Surg. 1994;9:662-72.

5. Griepp RB, Ergin MA, Galla JD, Klein JJ, Spielvogel D, Griepp EB. Minimizing spinal cord injury during repair of descending thoracic and thoracoabdominal aneurysms: the Mount Sinai approach. Semin Thorac Cardiovasc Surg. 1998;10:25-8.

6. Coselli JS, LeMaire SA, Miller CC III, Schmittling ZC, Koksoy C, Pagan J, et al. Mortality and paraplegia after thoracoabdominal aortic aneurysm repair: a risk factor analysis. Ann Thorac Surg. 2000;69: 409-14.

7. Terada H, Kazui T, Takinami M, Yamashita K, Washiyama N, Bashar AHM. Reduction of ischemic spinal cord injury by dextrorphan: comparison of several methods of administration. $J$ Thorac Cardiovasc Surg. 2001;122:979-85.

8. Shi E, Kazui T, Xiaojing J, Washiyama N, Suzuki K, Yamashita K, et al. NS-7, a novel $\mathrm{Na} / \mathrm{Ca} 2+$ channel blocker, prevents neurologic injury after spinal cord ischemia in rabbits. J Thorac Cardiovasc Surg. 2005; 129:364-71.

9. Liu D, Liu J, Sun D, Alcock NW, Wen J. Spinal cord injury increases iron levels: catalytic production of hydroxyl radicals. Free Radic Biol Med. 2003;34:64-71.

10. Mizuno A, Umemura K, Nakashima M. Inhibitory effect of MCI-186, free radical scavenger, on cerebral ischemia following rat middle cerebral artery occlusion. Gen Pharmac. 1998;30:575-8.
11. Edaravone Acute Infarction Study Group. Effect of a novel free radical scavenger, edaravone (MCI-186), on acute brain infarction. Randomized, placebo-controlled, double-blind study at multicenters. Cerebrovasc Dis. 2003;15:222-9.

12. Sakurai M, Hayashi T, Abe K, Aoki M, Sadahiro M, Tabayashi K. Enhancement of hear shock protein expression after transient ischemia in the preconditioned spinal cord of rabbits. J Vasc Surg. 1998;27: 720-5.

13. Kawai H, Nakai H, Suga M, Yuki S, Watanabe T, Saito K. Effects of a novel free radical scavenger, MCI-186, on ischemic brain damage in the rat distal middle cerebral artery occlusion model. J Pharmacol Exp Ther. 1997;271:921-7.

14. Takamatsu Y, Yuki S, Watanabe T. Studies on the concentrations of 3-methyl-1-phenyl-2-pyrazolin-5-one (MCI-186) in MCA occlusion and reperfusion model of rats. Jpn Pharmacol Ther. 1997;25(suppl): S1785-91.

15. Takamatsu Y, Watanabe T. Studies on the concentrations of 3-methyl1-phenyl-2-pyrazolin-5-one (MCI-186) in dog plasma and cerebral spinal fluid. Jpn Pharmacol Ther. 1997;25(suppl):S1793-7.

16. Setsukinai K, Urano Y, Kakinuma K, Majima HJ, Nagano T. Development of novel fluorescence probes that can reliably detect reactive oxygen species and distinguish specific species. J Biol Chem. 2003; 278:3170-5.

17. Johnson SH, Kraimer JM, Graeber GM. Effects of flunarizine on neurological recovery and spinal cord blood flow in experimental spinal cord ischemia in rabbits. Stroke. 1993;24:1547-53.

18. Takahashi G, Sakurai M, Abe K, Itoyama Y, Tabayashi K. MCI-186 prevents spinal cord damage and affects enzyme levels of nitric oxide synthase and $\mathrm{Cu} / \mathrm{Zn}$ superoxide dismutase after transient ischemia in rabbits. J Thorac Cardiovasc Surg. 2003;126:1461-6.

19. Lewen A, Matz P, Chan PH. Free radical pathways in CNS injury. J Neurotrauma. 2000;17:871-90.

20. Ikeda Y, Long DM. The molecular basis of brain injury and brain edema: the role of oxygen free radicals. Neurosurgery. 1990;27:1-11.

21. Floyd RA, Carney JM. Free radical damage to protein and DNA: mechanisms involved and relevant observations on brain undergoing oxidative stress. Ann Neurol. 1992;32(suppl):S22-7.

22. Traystman RJ, Kirsch JR, Koehler RC. Oxygen radical mechanisms of brain injury following ischemia and reperfusion. J Appl Physiol. 1991; 71:1185-95.

23. Sen S, Goldman H, Morehead M, Murphy S, Phillis JW. $\alpha$-Phenyltert-butyl-nitrone inhibits free radical release in brain concussion. Free Radic Biol Med. 1993;16:685-91.

24. Cao W, Carney JM, Duchon A, Floyd RA, Chevion M. Oxygen free radical involvement in ischemia and reperfusion injury to brain. $\mathrm{Neu}$ rosci Lett. 1988;88:233-8.

25. Hall ED, Andrus PK, Yonkers PA. Brain hydroxyl radical generation in acute experimental head injury. J Neurochem. 1993;60:588-94.

26. Bao F, Liu D. Hydroxyl radicals generated in the rat spinal cord at the level produced by impact injury induce cell death by necrosis and apoptosis: protection by a metalloporphyrin. Neuroscience. 2004;126: 285-95.

27. Yan EB, Unthank JK, Castillo-Melendez M, Miller SL, Langford SJ. Walker DW. A novel method for in vivo hydroxyl radical measurement by microdialysis in fetal sheep brain, in utero. J Appl Physiol. 2005;98:2304-10.

28. Sen S, Goldman H, Morehead M, Murphy S, Phillis JW. Oxypurinol inhibits free radical release from the cerebral cortex of closed head injured rats. Neurosci Lett. 1993;162:117-20.

29. Misso NLA, Peacock CD, Watkins DN, Thompson PJ. Nitrite generation and antioxidant effects during neutrophil apotosis. Free Radic Biol Med. 2000;28:934-43.

30. Watanabe K, Tanaka M. Chemical, pharmacological and clinical profile of a neuroprotective agent edaravone. Pharmacometrics. 2003;65: $79-88$. 